\title{
Reversed-phase Chromatography in an Extended Nanospace: Separating Amino Acids in Short and Long Nanochannels
}

\author{
Adelina Smirnova, Hisashi Shimizu, Kazuma Mawatari, and Takehiko KITAMori ${ }^{\dagger}$ \\ Department of Applied Chemistry, Graduate School of Engineering, The University of Tokyo, 7-3-1 Hongo, \\ Bunkyo, Tokyo 113-8656, Japan
}

\begin{abstract}
Micro- and nanofluidics has attracted much attention, particularly concerning single-cell analysis when small amounts of liquids are examined. In present work we successfully fabricated extended-nano channels that were more narrow and shorter $(2 \mathrm{~mm})$ as well as wider and longer $(10 \mathrm{~mm})$, and accomplished a reversed-phase HPLC separation of labeled amino acids on these channels after octadecylsilylation (ODS). The separation performance characteristics were compared for both types of nano spaces. At an equal amount of pressure, the longer extended-nano channels showed permeability that was one-order higher $\left(K=47 \times 10^{-14} \mathrm{~m}^{2}\right)$ and separation impedance $(E=13)$ that was one-order lower than that of the shorter version. Also, the separation plate number for the longer channel was 4000 with a plate height of $2.5 \mu \mathrm{m}$. Both channels have advantages for use in single-cell analysis. The longer channel can be applied for the separation of macromolecules (proteomics), while the short version is more applicable to small molecules (amino acids).
\end{abstract}

Keywords Extended-nano space, microchip, RP-HPLC, amino acids separation

(Received August 18, 2015; Accepted September 9, 2015; Published November 10, 2015)

\section{Introduction}

Reversed-phase high-performance liquid chromatography (RPHPLC) is an important technique for the analysis of complex biological samples (separation of omics, proteins, peptides, amino acids). RP-HPLC realized on a microscale allows one to study metabolism in an individual cell. ${ }^{1}$ Chromatographic separations in extended-nano space $\left(10^{1}-10^{2} \mathrm{~nm}\right.$ scale), developed by our group, ${ }^{2-5}$ can deal with sample volumes that range from attoliters to femtoliters with a high separation efficiency and a separation time of several seconds, and can be applied to single-cell analysis. Liquid chromatography in extended-nano space is based on "open tube" nanofluidics, wherein separation takes place in a single open tubular wallcoated nanochannel. This technique overcomes the limitations of a packed column due to the absence of packing material (the stationary phase is located on the channel walls), so that the influences of both Eddie diffusion and radial diffusion on the separation efficiency can be eliminated. Another way to obtain a much higher efficiency is to switch to a longer column. However, a simple increase in the channel length requires the growth of applied pressure as well, which is limited by the microchip stability. To obtain higher efficiency with the same pressure, flow velocity is gained by increasing the channel diameter.

The efficiency of chromatographic columns is determined by the plate numbers, $N$, as well as by the plate height, $H$, which is defined as the required length of a column in which a solute molecule equilibrates between the stationary and mobile phases.
For a rectangular cross-section, the extended-nano channel, $H$, can be expressed as follows:

$$
H=\frac{2 D_{\mathrm{m}}}{u}+\frac{f_{0} d^{2}}{105 D_{\mathrm{m}}} u
$$

where $D_{\mathrm{m}}$ is the molecular diffusion coefficient, $u$ is the flow velocity of the mobile phase, $f_{0}$ is a constant that is determined by the width-to-depth ratio of the separation channel, ${ }^{6}$ and $d$ is either the representative size of the nanochannel or the hydrodynamic diameter, $d=2 D W /(D+W)$, where $D$ and $W$ are the channel depth and width, respectively. The solvent velocity, $u$, is given by $u=K \Delta P / \eta L$, where $K$ is the permeability factor, $\Delta P$ is the net pressure drop over the chromatographic column, $\eta$ is the viscosity of the solvent, and $L$ is the length of the column. The solvent velocity and the viscosity in extended-nano channels can be estimated. ${ }^{7}$ The total resolving power of a column corresponds to the total $N$, which is calculated as $N=L / H$. Generally, more efficient columns have a smaller $H$ and a greater $N$.

Our group has already realized chromatographic separations on microchips with extended-nano channel lengths of $1 \mathrm{~mm}^{3,5}$ and $5 \mathrm{~mm}^{4}$ for the normal-phase mode and $2 \mathrm{~mm}$ for the reversephase mode. ${ }^{8}$ In the present work, we studied the influence of a lengthened and extended-nano channel on the efficiency of the reversed-phase separation of labeled amino acids. This technique can also be applied to unique single-cell analysis, which allows a determination of the content of intracellular amino acids.

$\dagger$ To whom correspondence should be addressed.

E-mail: kitamori@icl.t.u-tokyo.ac.jp 

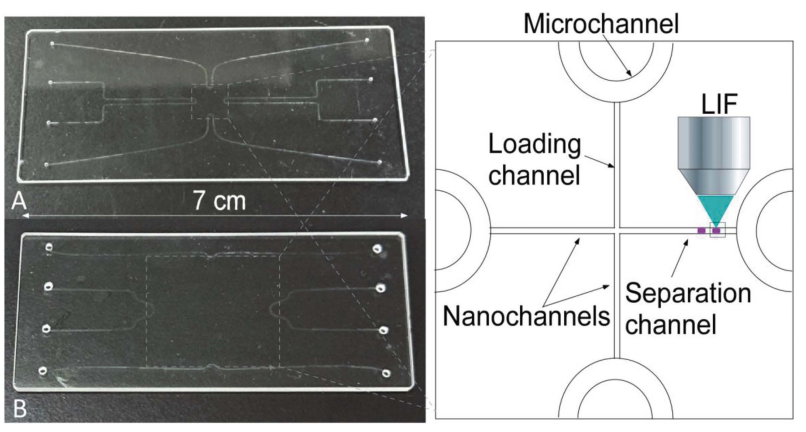

Fig. 1 Photograph of microchips (lengths of the nanochannels: A - $2 \mathrm{~mm}, \mathrm{~B}-10 \mathrm{~mm}$ ) with a close-up of the extended-nano channel design.

\section{Experimental}

\section{Reagents and chemicals}

Amino acids (L-serine, L-alanine, L-valine, and L-proline), sodium hydroxide, sodium tetraborate, citric acid, sodium perchlorate monohydrate and diethylamine were purchased from Wako Pure Chemical Industries, Ltd. (Osaka, Japan). The 4-Fluoro-7-nitro-2,1,3-benzoxadiazole (NBD-F) was obtained from Dojindo Laboratories (Kumamoto, Japan). Chloro(dimethyl)octadecylsilane, 95\% (ODS-Cl), was obtained from Sigma-Aldrich (St. Louis, MO). Toluene and acetonitrile were of HPLC grade and were obtained from the Kanto Chemical Co., Inc. (Tokyo, Japan). Water was purified using a Milli-Q system (Millipore, Bedford, MA).

\section{Chip fabrication and design}

The extended-nano channels were fabricated on a quartz glass plate using electron-beam lithography (ELS-7500, Elionix, Tokyo, Japan) and plasma etching, ${ }^{9}$ and the microchannels were fabricated from the same substrate via mask alignment and plasma etching. All fabrication procedures were performed in class 100 and 1000 clean rooms. The substrates with channels and cover plates were bonded using a high-temperature bonding technique $\left(1000^{\circ} \mathrm{C}\right)$. Under high-temperature, hydrophobic strained siloxane, $\mathrm{Si}-\mathrm{O}-\mathrm{Si}$ bonds were formed on the surface. In order to recover any free silanol groups, the channels were washed with a $0.1 \mathrm{M}$ sodium hydroxide solution for $30 \mathrm{~min}$ before being rinsed with water. The design of the nanochannels consisted of loading, separation, and waste channels with a crossed shape (Fig. 1). ${ }^{5}$ The four ends of the nanochannels were linked to microchannels. Each end of the microchannels was connected to a custom-made pressure-driven system. ${ }^{10}$ The width and depth of a $2 \mathrm{~mm}$ length of the nanochannel measured 800 and $230 \mathrm{~nm}$, and the width and depth of a $10 \mathrm{~mm}$ length of nanochannel measured 2000 and $470 \mathrm{~nm}$, respectively. The microchannels were $250 \mu \mathrm{m}$ wide and $3 \mu \mathrm{m}$ deep.

\section{Chromatographic conditions}

Pressure-driven pinched injection was performed as previously described, ${ }^{10}$ with the only difference being the use of a nitrogen gas cylinder to provide pressure as high as $10 \mathrm{MPa}$ instead of a compressor, which was limited to $3.5 \mathrm{MPa}$. For sample loading, pressure was applied to the 3 microchannels from both ends. During sample injection, pressure from the right side of the separation channel was switched off first, and when the pressure from the loading channel was switched off the band injection was completed. Timing control was performed with a time resolution of $10 \mathrm{~ms}^{2,10}$

A microchip was placed in a custom-made chip holder, which was then fixed on a motorized stage (BIOS 105T, Sigma Kouki Co., Ltd., Tokyo, Japan) of a fluorescence microscope (IX71, Olympus, Tokyo, Japan). For signal generation, we used laserinduced fluorescence (LIF) with an excitation at $488 \mathrm{~nm}$ (Coherent Sapphire 488-500 CDRH, Germany, Olympus WIB cube filter: BP 460-490, BA 515IF, Olympus, Tokyo, Japan), and for fluorescence detection and signal analysis we used a CCD camera (ImageEM, Hamamatsu Photonics K.K., Shizuoka, Japan) and Aquacosmos software (Hamamatsu, Photonics K.K., Shizuoka, Japan).

The mobile phase consisted of $25 \mathrm{mM}$ citrate buffer and $25 \mathrm{mM}$ sodium perchlorate ( $\mathrm{pH} 5.5)$ /acetonitrile $(92 / 8, \mathrm{v} / \mathrm{v})$. All experiments were performed at room temperature, $25^{\circ} \mathrm{C}$. The mix of serine, alanine, proline, and valine was derivatized by NBD-F, according to a method described by Song. ${ }^{11}$ An NBDF-labeled amino acids mix was then dissolved in the mobile phase (buffer/acetonitrile 70/30, v/v) to bring the working concentration to $25 \mu \mathrm{M}$.

To realize reversed-phase HPLC mode separation, the walls of the extended-nano channels were modified with $\mathrm{C} 18$. For the modification we used octadecyldimethyl- $N, N$-diethyiaminosilane (ODS-DEA) synthesized from diethylamine and ODS-Cl, according to a method proposed by Tanaka. ${ }^{12}$ For the modification procedure, acetonitrile, dried toluene, and $20 \%$ $(\mathrm{v} / \mathrm{v})$ ODS-DEA in toluene (at $50^{\circ} \mathrm{C}$ ) were continuously introduced over a period of $2 \mathrm{~h}$ into one microchannel, which filled the nanochannels for $30 \mathrm{~min}$ each. Afterward, all microchannels again were rinsed with toluene and acetonitrile.

\section{Results and Discussion}

We estimated the peak capacity for separation in the $2 \mathrm{~mm}$ extended-nano channels according to the following formula:

$$
n=1+\frac{\sqrt{N}}{4} \ln \frac{V_{\max }}{V_{\min }}
$$

where $N$ is the plate numbers and $V$ is the separation volume (proportional to retention time). This estimation showed that the maximum number of possible separated peaks was about $7-12$ when $N=300-600$ and $V_{\max } / V_{\min }=4-5$. To increase the capacity of a column, the nanochannel length was increased. However, a simple length increase also required a pressure increase. A value of $6 \mathrm{MPa}$ for separation almost reaches the limits for microchip stability. We estimated the effect of the channel length increase on the separation efficiency with and without increasing the channel hydrodynamic diameter. All calculations were performed according to Eq. (1), where $D_{\mathrm{m}}=1 \times 10^{-9} \mathrm{~m}^{2} / \mathrm{s}, f_{0}=5.36 ;{ }^{6}$ there would also be a constant pressure drop. This calculation showed that a simple lengthening of the separation distance at the same $d$ and pressure value provides no advantages in the separation efficiency, and remaining $N$ unchanged. To improve the performance characteristics, the representative size of nanochannel $d$ must be increased along with the length of the extended-nano channel.

The new chip was fabricated according to new specifications. For both types of chips we separated the derivatized amino acids mix using the same experimental conditions (mobile phase, applied pressure, detection system) (Fig. 2). The characteristics of the separation and the elution order of the amino acids were similar for chips with both short and long extended-nano 


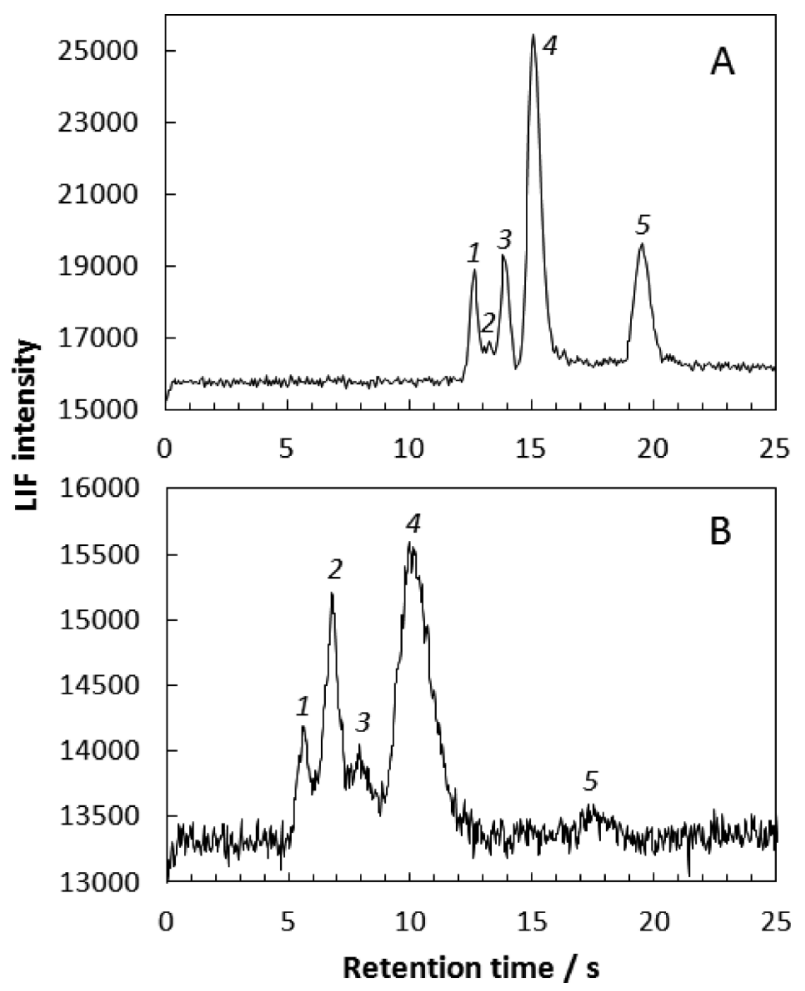

Fig. 2 Chromatograms obtained for the NBD-F derivatives of amino acids ( 1 - serine, 2 - NBD-OH, 3 - alanine, 4 - proline, 5 - valine) for extended-nano channels. The channel lengths were as follows: A $-10 \mathrm{~mm}, \mathrm{~B}-2 \mathrm{~mm}$. The mobile phases were as follows: $25 \mathrm{mM}$ Citric buffer (pH 5.5), $25 \mathrm{mM}$ sodium perchloride, acetonitrile (buffer/ acetonitrile $-92 / 8), c_{\text {amino acid }}=25 \mu \mathrm{M}$.

Table 1 Comparison of the separation characteristics of the NBD-F derivatives of amino acids with both short and long nanochannel extension

\begin{tabular}{lrrccc}
\hline & Length/mm & serine & alanine & proline & valine \\
\hline$N$, plate numbers & 2 & 320 & 329 & 2269 & 212 \\
& 10 & 4030 & 3800 & 1890 & 3100 \\
$N / m$, Plates $/ \mathrm{m}$ & 2 & $160 \mathrm{~K}$ & $165 \mathrm{~K}$ & $113 \mathrm{~K}$ & $106 \mathrm{~K}$ \\
& 10 & $400 \mathrm{~K}$ & $380 \mathrm{~K}$ & $190 \mathrm{~K}$ & $310 \mathrm{~K}$ \\
$H$, Plate height $\mu \mathrm{m}$ & 2 & 6.2 & 6.1 & 8.8 & 9.4 \\
& 10 & 2.5 & 2.6 & 5.3 & 3.2 \\
\hline
\end{tabular}

$\mathrm{K}=1000$.

channels. The height of the second peak of NBD-OH depended on the excess of NBD-F during the labeling process. The separation characteristics are summarized in Table 1. As predicted, we could increase $N$ by one order by changing the extended-nano channel length and the diameter without increasing the applied pressure. The peak capacity for the $10 \mathrm{~mm}$ extended-nano channel became $14-18$, which was twice the capacity of the $2 \mathrm{~mm}$ channel.

For a comparison of the performance aspects of the two columns (narrow-short and wide-long), we used "separation impedance", which is a concept that was introduced by Knox and Bristow, ${ }^{13,14}$ which describes the column design as a compromise between the speed-efficiency-pressure and the time required for plate generation:

$$
E=h^{2} K=\frac{t_{0} \Delta P}{N^{2} \eta}
$$

Here, $E$ is the separation impedance and $h$ is the reduced $H$. Based on the results of capillary filling experiments, ${ }^{7}$ we estimated the separation impedance and permeability for a $2 \mathrm{~mm}$ length of nanochannel to be 580 and $5-6 \times 10^{-14} \mathrm{~m}^{2}$, respectively. For a $10 \mathrm{~mm}$ channel, $E$ and $K$ became 13 and $47 \times 10^{-14} \mathrm{~m}^{2}$, respectively. This showed that the separation impedance strongly varied with the retention factor, which meant that a longer and wider channel showed better performance with a separation efficiency that was one-order higher than that of a shorter and more narrow channel under the same pressure conditions.

\section{Conclusions}

We succeeded to separate a 30 aL-labeled amino acid mixture with a high $N\left(2-4 \times 10^{3}\right.$ plates $)$ and a fast separation time (20 s) by using a nano channel that had been extended to $10 \mathrm{~mm}$ in length. The longer nanochannel showed better separation efficiency compared with the shorter one under the same amount of applied pressure: a one-order higher $N$ and a 3-fold lower $H$; also, a one-order higher permeability and a one-order lower separation impedance. Extended-nano channels that are longer and wider can be applied in cases calling for more complicated and larger substances, such as proteins and metabolites. After integration with a single-cell capture technique and cell lysis, ${ }^{15}$ it would be possible to study both the content of a single cell as well as cell-to-cell variability and cell-to-cell communications in real time.

\section{Acknowledgements}

We are grateful to Prof. Shigeru Terabe for fruitful discussions and helpful advice, and we are thankful for the financial support from CREST (Japan).

\section{References}

1. K. Kleparnik and F. Foret, Anal. Chim. Acta, 2013, 800, 12.

2. M. Kato, M. Inaba, T. Tsukahara, K. Mawatari, A. Hibara, and T. Kitamori, Anal. Chem., 2010, 82, 543.

3. H. Shimizu, K. Mawatari, and T. Kitamori, Analyst, 2014, 139, 2154.

4. R. Ishibashi, K. Mawatari, and T. Kitamori, J. Chromatogr. A, 2012, 1238, 152.

5. R. Ishibashi, K. Mawatari, and T. Kitamori, Small, 2012, 8 , 1237.

6. H. Poppe, J. Chromatogr. A, 2002, 948, 3.

7. L. X. Li, Y. Kazoe, K. Mawatari, Y. Sugii, and T. Kitamori, J. Phys. Chem. Lett., 2012, 3, 2447.

8. A. Smirnova, H. Shimizu, K. Mawatari, and T. Kitamori, $J$. Chromatogr. A, 2015, in press.

9. T. Tsukahara, T. Kuwahata, A. Hibara, H. B. Kim, K. Mawatari, and T. Kitamori, Electrophoresis, 2009, 30, 3212.

10. R. Ishibashi, K. Mawatari, K. Takahashi, and T. Kitamori, J. Chromatogr. A, 2012, 1228, 51.

11. Y. T. Song, T. Funatsu, and M. Tsunoda, J. Chromatogr. B, 2011, 879, 335.

12. N. Tanaka, H. Kinoshita, M. Araki, and T. Tsuda, $J$. 
Chromatogr., 1985, 332, 57.

13. J. H. Knox and M. T. Gilbert, J. Chromatogr., 1979, 186, 405.

14. P. A. Bristow and J. H. Knox, Chromatographia, 1977, 10 , 279.
15. L. Lin, K. Mawatari, K. Morikawa, and T. Kitamori, Proceedings of the 19th International Conference on Miniaturized Systems for Chemistry and Life Sciences ( $\mu$ TAS2015), ed. J.-K. Park, 2015, Gyeongju, Korea, in press. 